\title{
Epithelium-Specific Ets-Like Transcription Factor 1, ESE-1, Regulates ICAM-1 Expression in Cultured Lung Epithelial Cell Lines
}

\author{
Zhiqi Yu, ${ }^{1}$ Jun Xu, ${ }^{1}$ Jinbao Liu, ${ }^{2}$ Jing Wu, ${ }^{3}$ Chan Mi Lee, ${ }^{3,4}$ Li Yu, ${ }^{5}$ and Jim Hu ${ }^{3,4}$ \\ ${ }^{1}$ State Key Lab of Respiratory Disease and Guangzhou Institute of Respiratory Disease, \\ The First Affiliated Hospital of Guangzhou Medical University, Guangzhou Medical University, Guangzhou 510120, China \\ ${ }^{2}$ Protein Modification and Degradation Laboratory, Department of Pathophysiology, \\ Guangzhou Medical University, Guangdong, China \\ ${ }^{3}$ Physiology \& Experimental Medicine Program, Hospital for Sick Children, Toronto, ON, Canada M5G 1X8 \\ ${ }^{4}$ Department of Laboratory Medicine and Pathobiology, University of Toronto, Toronto, ON, Canada M5S 1A8 \\ ${ }^{5}$ Department of Pediatrics, Guangzhou First People's Hospital, Affiliated to Guangzhou Medical University, \\ Guangzhou, Guangdong 510180, China
}

Correspondence should be addressed to Jim Hu; jim.hu@utoronto.ca

Received 14 November 2014; Revised 31 December 2014; Accepted 5 January 2015

Academic Editor: Marcus A. Mall

Copyright (c) 2015 Zhiqi Yu et al. This is an open access article distributed under the Creative Commons Attribution License, which permits unrestricted use, distribution, and reproduction in any medium, provided the original work is properly cited.

Cystic fibrosis (CF) patients suffer from chronic airway inflammation with excessive neutrophil infiltration. Migration of neutrophils to the lung requires chemokine and cytokine signaling as well as cell adhesion molecules, such as intercellular adhesion molecule-1 (ICAM-1), which plays an important role in mediating adhesive interactions between effector and target cells in the immune system. In this study, we investigated the relationship between ICAM-1 and epithelium-specific ETS-like transcription factor 1 (ESE-1) and found that ICAM-1 expression is upregulated in cell lines of CF (IB3-1) as well as non-CF (BEAS-2B and A549) epithelial origin in response to inflammatory cytokine stimulation. Since ESE-1 is highly expressed in A549 cells without stimulation, we examined the effect of ESE-1 knockdown on ICAM-1 expression in these cells. We found that ICAM-1 expression was downregulated when ESE-1 was knocked down in A549 cells. We also tested the effect of ESE-1 knockdown on cell-cell interactions and demonstrate that the knocking down ESE-1 in A549 cells reduce their interactions with HL-60 cells (human promyelocytic leukemia cell line). These results suggest that ESE-1 may play a role in regulating airway inflammation by regulating ICAM-1 expression.

\section{Introduction}

Airway inflammation is a hallmark of the cystic fibrosis (CF) lung disease. The airways of CF patients are initially colonized by viruses, fungi, or bacteria, including Staphylococcus aureus, Haemophilus influenzae, and Klebsiella pneumonia [1]. Most patients later become infected with mucoid strains of Pseudomonas aeruginosa and some with Burkholderia cepacia [2].

In $\mathrm{CF}$ patients, the number of neutrophils and the levels of cytokines such as tumor necrosis factor- $\alpha$ (TNF- $\alpha$ ), interleukin- (IL-) 6 , and IL-8 in the airways are increased compared to non-CF individuals $[3,4]$. Cultured CF lung epithelial cells (IB3-1) show downregulation of the antiinflammatory cytokine IL-10 and an exaggerated upregulation of IL-8 in response to a variety of external stimuli, such as TNF- $\alpha$ and bacterial products $[5,6]$. Overproduction of IL8 is likely a major cause of excessive neutrophil infiltration, since IL-8 is a potent chemoattractant for neutrophils [7].

Neutrophil migration in response to inflammatory stimuli requires cell adhesion molecules, such as intercellular adhesion molecule-1 (ICAM-1, also known as CD54) [8, 9]. Migration of neutrophils out of the vascular system occurs in distinct phases: rolling, firm adhesion, and transmigration [10]. 
Four types of cell adhesion molecules are involved in this process, namely, E-selectin, ICAM-1, vascular cell adhesion molecule-1 (VCAM-1), and platelet-endothelial cell adhesion molecule-1 (PECAM-1). Neutrophil rolling is the first step of the migration process and E-selectin is the key molecule involved in slowing down the circulating neutrophils. This step is critical to ensure firm adhesion of neutrophils to the endothelial cell layer. Firm adhesion is mediated through ICAM-1 expressed on endothelial cells, which interacts with CD11a/CD18 (LFA1) or CD11b/CD18 (Mac-1) as counterreceptors on neutrophils [11]. The final phase of transmigration of neutrophils through the endothelium is triggered by PECAM-1 and VCAM-1 [10]. Currently, the mechanism by which neutrophils migrate to the airway lumen is unclear, but they are thought to travel through the intercellular space $[12,13]$. Other cell adhesion molecules such as ICAM-2 and ICAM-3 are also involved in the migration of monocytes [14] or dendritic cells [15].

ICAM-1 is a $114 \mathrm{kD}$ inducible surface glycoprotein that belongs to the immunoglobulin superfamily [9] and it plays an important role in innate and adaptive immune responses [16]. Although the role of ICAM-1 in endothelial cells as well as in adaptive immunity [17-20] is well established, the function of epithelial ICAM-1 during inflammation is not fully understood. Since epithelial ICAM-1 is expressed on the airway lumen [21-24], a role for leukocyte transmigration is not expected. On the other hand, cell adhesion studies [25, 26] indicate that epithelial ICAM-1 is important for leukocyte homing. Because neutrophils and macrophages are enriched at the sites of injury or inflammation, it is possible that homing of these cells is part of the resolution of inflammation.

Among the adhesion molecules, ICAM-1 may play a more important role in the infiltration of leukocytes during airway inflammation. For example, Hubeau et al. performed quantitative analysis of inflammatory cells infiltrating the CF airway mucosa in lung tissues collected at the time of transplantation and found that ICAM-1, but not VCAM-1 or E-selectin, was overexpressed on the epithelium surface [27]. In addition, a recent in vitro study also showed that ICAM-1 is expressed in a higher percentage of cultured airway epithelial cell lines (IB3-1, C38 and BEAS-2B) than other cell adhesion molecules, such as VCAM-1 or E-selectin [28].

ICAM-1 is expressed at a very low level in airway epithelial cells. Interestingly, CF-deficient airway epithelial cells have a slightly higher basal level of ICAM-1 expression [28]. Upon stimulation with proinflammatory cytokine (e.g., TNF- $\alpha$ or IL-1 $\beta)[29,30]$ or other stimulatory substances (e.g., LPS) [31], ICAM-1 expression is significantly induced in both human primary bronchial epithelial cultures and epithelial cell lines. This induction is mediated by activation of nuclear factor-kappa B (NF- $\kappa \mathrm{B})$ signaling transduction pathway. In addition, ICAM- 1 induction can also be mediated through the STAT signaling pathway since IFN-gamma can significantly elevate its expression in epithelial cells [16]. CF airways have chronic inflammation, which contributes to the overexpression of ICAM-1 [27]. Since epithelial ICAM-1 may be critical for neutrophil homing and epithelial killing, it is important to understand its regulation and function in airway epithelial cells in order to identify potential drug targets for the CF lung disease.

The E26 transformation-specific (ETS) family of transcription factors is characterized by a highly conserved 85 amino acid DNA binding domain, which is known as the ETS domain [32]. It is comprised of 27 and 26 members in humans and mice, respectively. The ETS domain is usually located in the carboxyl-terminal region of the protein as a winged helix-turn-helix structural motif and binds to purine-rich DNA that has a core consensus sequence of GGAA/T- within the promoter and enhancer regions of target genes [33]. ETS transcriptional factors act as both positive and negative regulators of gene expression in various biological processes, such as cellular proliferation, differentiation, apoptosis, metastasis, hematopoiesis, and angiogenesis [34]. Although many of ETS family members are expressed in nonepithelial cells, such as hematopoietic and endothelial cells, ESE-1 is mainly expressed in epithelial-rich tissues, such as lung, kidney, stomach, small intestine, colon, pancreas, trachea, salivary gland, prostate gland, mammary gland, uterus, and skin [35], but it can be upregulated in nonepithelial cells by proinflammatory cytokines such as TNF- $\alpha$ and IL- $1 \beta$. Our previous work has shown that ESE-1 can be highly induced in epithelial cells by inflammatory cytokines [36].

In this study, we investigated the regulation of ICAM-1 expression by ESE-1. Here we demonstrate that the expression of both ICAM-1 and ESE-1 is upregulated in human bronchial epithelial cells (BEAS2B), CF cells (IB3-1), and lung cancer cells (A549) by inflammatory cytokines. We also show that ICAM-1 expression is downregulated upon ESE-1 knockdown in A549 cells and that ESE-1 regulates the ICAM-1 expression at the transcriptional level. Finally, we demonstrate that the downregulation of ICAM-1 by knocking down ESE-1 in A549 cells results in a reduced capacity of A549 cells to interact with HL-60 cells.

\section{Materials and Methods}

2.1. Cell Culture and Reagents. Human bronchial epithelial cells (BEAS-2B), human lung carcinoma cells (A549), and CF bronchi epithelial cells (IB3-1) were cultured in Dulbecco's Modified Eagle's Medium (DMEM) medium (Invitrogen) supplemented with $10 \%$ fetal bovine serum (FBS). Recombinant human tumor necrosis factor- $\alpha$ (TNF- $\alpha$ ) and interleukin-1 $\beta$ (IL-1 $\beta$ ) were obtained from R\&D Systems (Minneapolis, $\mathrm{MN}$ ) and reconstituted in phosphate buffered saline (PBS) containing $0.1 \%$ bovine serum albumin (BSA). For cytokines stimulation, TNF- $\alpha$ and IL- $1 \beta$ were used at $10 \mathrm{ng} / \mathrm{mL}$ each.

\subsection{Viral Transduction and shRNA-Mediated Gene Knocking} Down. ESE-1 gene was knocked down in A549 cells using a shRNA helper-dependent adenoviral vector expressing two shRNAs from murine U6 gene promoter as described previously [36]. A C4HSU empty vector was used as control. For viral transduction, A549 cells were seeded at $2 \times 10^{5}$ cells per well in 6-well plates overnight, and then cells were transduced at $40-60 \%$ confluency with viral vector at 
2500 particles/cell (or 50 moi) under serum-free conditions for two hours, followed by addition of media to a final concentration of $10 \%$ FBS. We usually achieved near $100 \%$ transduction at this vector concentration. Cells were then collected for protein extraction or RNA isolation at desired time points.

2.3. Transient Transfection and Luciferase Reporter Assay. For cell transfection and cotransfection experiments, BEAS-2B cells were seeded in 6-well plates and transfected at 50-60\% confluency by using PolyFect transfection reagent (Qiagen) according to the manufacturer's protocol. After $24 \mathrm{~h}$, cells were harvested and luciferase activity was measured using a Dual Lucifcerase kit (Promega) and a luminometer (EG\&G Berthold, BadWildbad, Germany) as described previously [37].

2.4. RNA Analysis. Cells were lysated and RNAs were extracted by using RNA spin Mini kit (GE Healthcare) according to manufacturer's instructions. Total RNA $(1 \mu \mathrm{g})$ was reverse transcribed using random hexamers and SuperScript II reverse transcriptase (Invitrogen), and then the resulting template (20 ng cDNA) was used for each real-time PCR reaction (ABI Prism 7700, Applied Biosystems). Primers for human ESE-1 and ICAM-1 were purchased from ACGT, Toronto. For relative quantification, PCR signals were compared between groups after normalization with GAPDH as an internal reference. Fold changes were calculated according to Livak and Schmittgen [38]. Primers used were hICAM1F: GGAAGGTGTATGAACTGAGCAA, hICAM1-R: GGAGTCCAGTACACGGTGA, hesel-F: GGCGTCTTCAAGTTCCTGCG, hesel-R: CTCCCGTTTGTAGTAGTACCT, hGAPDH-F: GAAGGTGAAGGTCGGAGTC, hGAPDH-R: GAAGATGGTGATGGGATTTC.

2.5. Western Blotting Analysis. Cell lysates were run on an $8 \%$ SDS-polyacrylamide gel at $10 \mu \mathrm{g}$ each lane and transferred to nitrocellulose membrane (Bio-Rad) following electrophoresis. The membrane was then blocked with Tris-buffered saline with Tween-20 (TBST) containing 5\% milk and probed with goat anti-human ICAM-1 antibody (R\&D System, Minneapolis, USA) at 1:1000 dilution and rabbit anti-human ESE-1 antibody (R\&D System, Minneapolis, USA) at 1:5000 dilution. Rabbit anti-human GAPDH antibodies (Trevigen, Gaithersburg, USA) were used at 1:3000 as a protein loading control. The horseradish peroxidase-conjugated secondary antibodies for rabbit anti-goat IgG were from Kirkegaard \& Perry Laboratories and for goat anti-rabbit IgG were from Bio-Rad Laboratories. Detection of proteins was performed with enhanced chemiluminescence reagents (Amersham Pharmacia Biotech, Baie-d'Urfe, CA).

2.6. Cell Adhesion Assay. At day $0,1 \times 10^{6}$ A549 cells were seeded on a $10 \mathrm{~cm}$ dish and virus vector particles were added at 100 moi (5000 particles per cell) for 1 hour in serum-free media. Fetal bovine serum was then added to final concentration of $10 \%$. At day 5 , the treated cells were harvested and seeded at 250,000 to each well of a-24-well plate. The reason for waiting for 5 days was to minimize the influence of inflammatory cytokines induced by the viral transduction. For visualization of cell-cell interactions, HL60 cells were labeled with carboxyfluorescein succinimide ester (CFSE) at $5 \mu \mathrm{M}$ in the cell culture for $5 \mathrm{~min}$ at $37^{\circ} \mathrm{C}$ and then washed 3 times with RPMI 1640. The labeled HL- 60 cells were put back in the cell culture condition for 24 hours. The A549 cells were then stimulated with IL- $1 \beta$ and TNF- $\alpha$ each at $10 \mathrm{ng} / \mathrm{mL}$ for 6 hours and labeled HL- 60 cells were activated with 12-O-tetradecanoylphorbol-13-acetate (TPA) at $5 \mathrm{ng} / \mathrm{mL}$ for 4 hours. After washing both cells, 1 million HL-60 cells in $0.5 \mathrm{~mL}$ of RPMI 1640 were added to each well of the treated A549 cells under cell culture conditions. Following adhesion for 1 hour, nonattached HL-60 cells were washed away with 1x PBS for 3 times at room temperature. Cells were fixed with $1 \%$ Paraformaldehyde in 1x PBS for $10 \mathrm{~min}$ and washed 3 times with $1 \mathrm{x}$ PBS. Cells were photographed using a Leica Fluorescence Microscope (model, DMIRE2).

2.7. Statistical Analysis. Data were analyzed by using MannWhitney $U$ test (Kruskal-Wallis test for more than two groups). $P<0.05$ was considered statistically significant.

\section{Results}

3.1. ESE-1 and ICAM-1 Expression Are Upregulated in BEAS2B, IB3-1, and A549 Cells in Response to Inflammatory Cytokines. To examine whether both ESE-1 and ICAM-1 are upregulated under proinflammatory conditions, BEAS-2B, IB3-1, and A549 cells were stimulated with a combination of proinflammatory cytokines TNF- $\alpha$ and IL-1 $\beta$ (10 ng/mL each) for 2 hours, and their mRNA levels were assessed by qRT-PCR. As shown in Figures 1(a) and 1(b), levels of mRNA for both ESE-1 and ICAM-1 were increased significantly (except for ESE-1 expression in A549 cells). We also observed a higher level of ESE-1 mRNA expression in A549 cells than BEAS$2 \mathrm{~B}$ and IB3-1 cells. To further investigate at which time point ESE-1 and ICAM-1 start responding to cytokine stimulation, we completed a time course study. As shown in Figures 1(c) and 1(d), the ESE-1 mRNA expression peaked at 2 hours after stimulation in A549 cells while ICAM-1 mRNA expression reached a plateau at 4 hours after stimulation.

3.2. ICAM-1 Expression Is Downregulated by the ESE-1 Knockdown in A549 Cells. Since both ESE-1 and ICAM1 mRNA expression were upregulated when stimulated by proinflammatory cytokines and given that ESE-1 is a transcription factor, we questioned whether ICAM-1 expression is transcriptionally regulated by ESE-1. We used a helperdependent adenovirus (HD-Ad) vector to knockdown ESE1 gene expression in A549 cells and examined the changes in ICAM-1 expression. We selected A549 cells for the knockdown experiment, as these cells constitutively express relatively high levels of ESE-1, at both mRNA and protein levels. As expected, we found a significant reduction of ESE-1 mRNA following HD-Ad vector transduction (Figure 2(b)). Interestingly, ICAM-1 expression was also downregulated compared to the empty vector (C4HSU) control group 

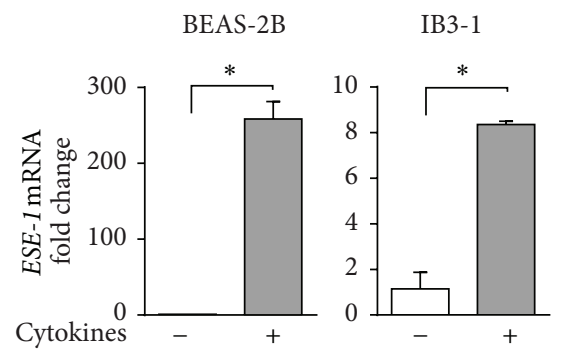

(a)

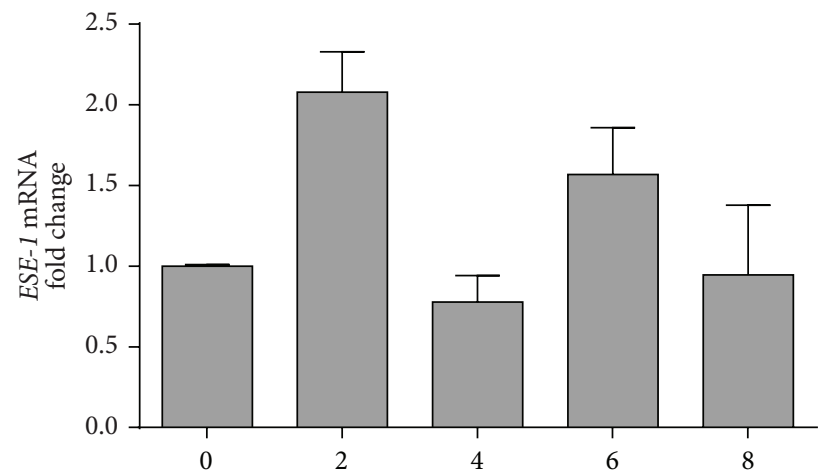

(h)

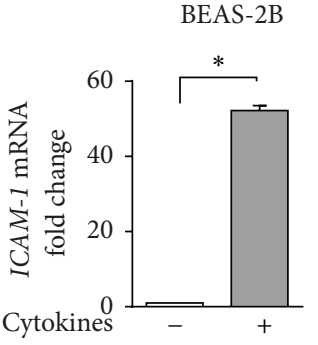

2500
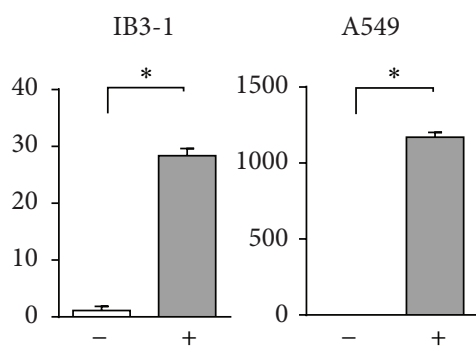

(b)

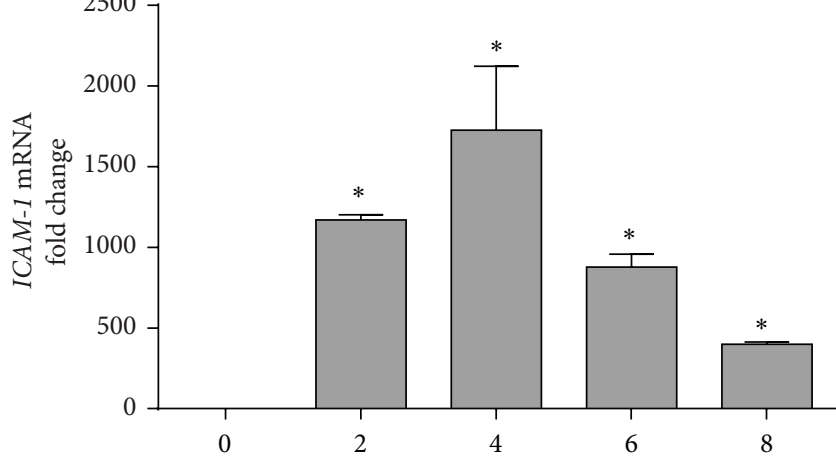

(h)

(c)

(d)

Figure 1: Induction of ESE-1 and ICAM-1 mRNA expression by TNF- $\alpha$ and IL-1 $\beta$ in different cell lines. (a, b) ESE- 1 and ICAM-1 mRNA expression in BEAS2B, IB3-1, and A549 cells after stimulation with TNF- $\alpha$ and IL-1 $\beta$ (10 ng/mL each) for 2 hours. The mRNA expression levels were determined by real-time quantitative RT-PCR and the fold of change was based on the mRNA level of non-induced cells. Data were normalized to GAPDH and values were shown in $2^{-\triangle \Delta C T}$ as the mean $\pm \mathrm{SD}, n=3,{ }^{*} P<0.05$. Statistics was performed as described in Materials and Methods. (c, d) ESE-1 and ICAM-1 mRNA expression in A549 cells at different time points following cytokine stimulation. Cells were lysed at time points as indicated after TNF- $\alpha$ and IL-1 $\beta(10 \mathrm{ng} / \mathrm{mL}$ each) stimulation. The fold of change was based on the expression level at time 0 hour. Data were collected and analyzed as described in (a) and (b).

(Figure 2(c)). We examined ESE-1 and ICAM-1 expression at days 4 and 5 after transduction because viral vectors alone can induce the production of proinflammatory cytokines that can last for more than two days after viral transduction. We also used longer time points for cytokine stimulation in protein analysis (16 h) than in mRNA analysis $(2 \mathrm{~h})$, as changes in protein expression lag that of the mRNA.

3.3. ESE-1 Gene Regulates ICAM-1 Expression at the Transcriptional Level. To further investigate the regulatory mechanism, we developed an ICAM-1 promoter reporter assay to examine the role of ESE-1 in the regulation of ICAM-1 expression. As shown in Figure 3(a), we made the reporter construct by inserting a $1.4 \mathrm{~kb}$ human ICAM-1 promoter in front of the luciferase reporter gene in pGL-3-basic vector (Promega). BEAS-2B cells were cotransfected with the ICAM-1 promoter luciferase reporter plasmid and pcDNA3ESE-1 or pcDNA3-empty as an empty vector control. BEAS2B cells are noncancerous human bronchial epithelial cells which express minimal basal levels of ESE-1, allowing us to specifically overexpress the protein by transfection. Twentyfour hours after cotransfection, the luciferase activity was measured and as shown in Figure 3(b), the level of luciferase activity was increased in the ESE-1 overexpression group compared to empty vector control group, suggesting that ESE-1 regulates ICAM-1 expression at the transcriptional level.

3.4. Knockdown of ESE-1 Expression in A549 Cells Reduces Binding of HL-60 Cells. Since ICAM-1 is involved in cellcell interactions, we performed a cell adhesion assay to investigate whether knockdown of ESE-1 gene could affect cellular adhesion. We found that, following transduction with ESE-1-RNAi viral vector, A549 cells had less binding of HL-60 cells compared with C4HSU empty vector transduced group (Figure 4).

\section{Discussion}

In response to environmental perturbations, airway epithelia produce and release a variety of inflammatory cytokines, including TNF- $\alpha$, IL-1, IL-6, and IL-8 [39]. A number of studies have shown that the epithelium of patients who have airway inflammatory diseases is structurally and functionally altered, and that bronchial epithelial cells that are isolated from patients with asthma or cystic fibrosis (CF) express increased levels of cytokines (IL-8, IL-25) [40, 41]. It is thus important to fully understand the gene regulation in 


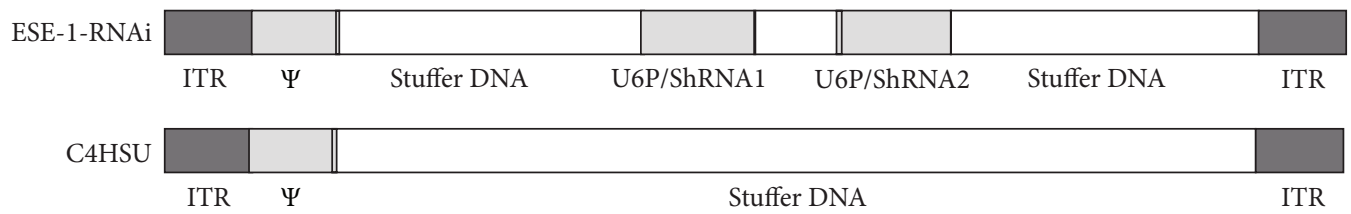

(a)

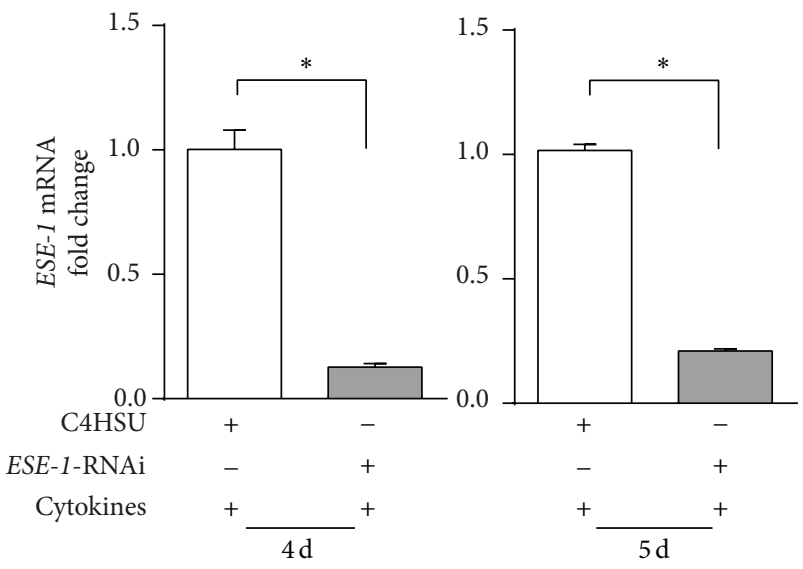

(b)
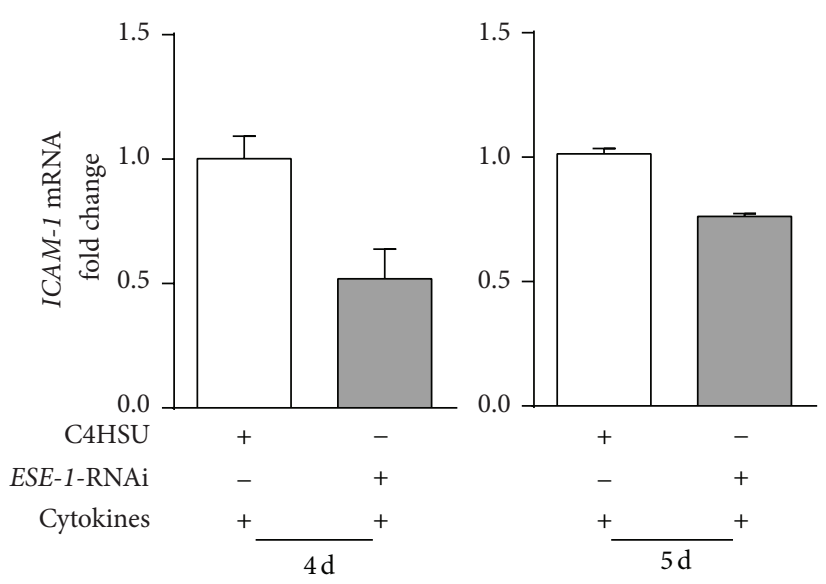

(c)

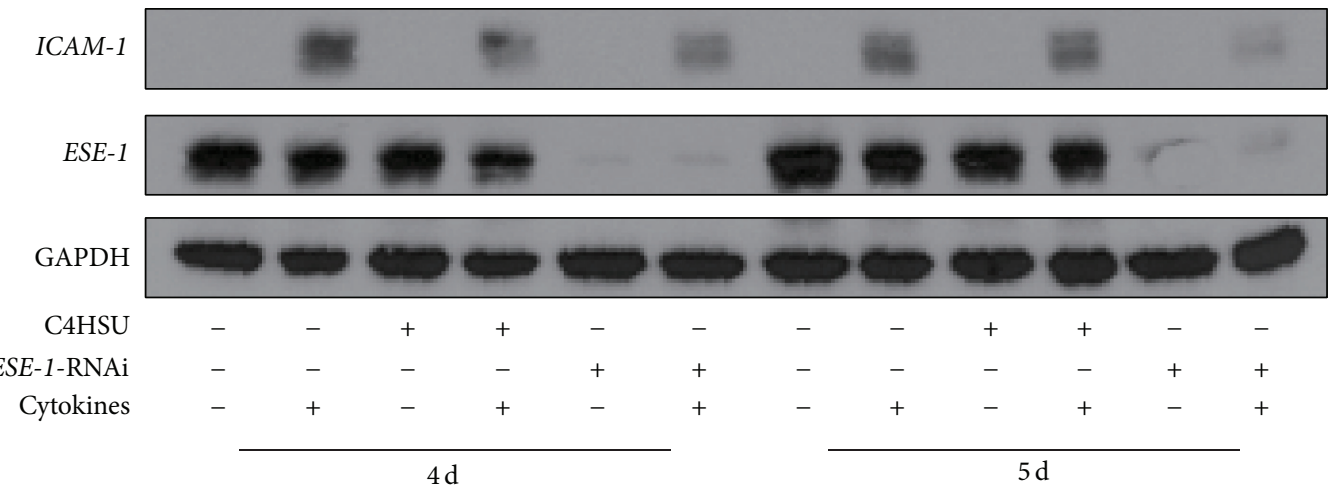

(d)

FiguRE 2: Effects of ESE-1 knockdown in A549 cells on the expression of ICAM-1. (a) Schematic diagrams of the helper-dependent adenovirus vectors (HD-Ad) that were used for the ESE-1 knockdown experiments. ESE-1-RNAi expresses two shRNAs from the murine U6 gene promoter, and C4HSU is used as an empty vector control which does not express any transgene. ITR: inverted terminal repeat; $\Psi$ : packing signal. (b, c) ESE-1 and ICAM-1 mRNA expression in A549 cells 4 and 5 days after transduction with ESE-1-RNAi vector compared to that of C4HSU. Both groups were stimulated with TNF- $\alpha$ and IL- $1 \beta$ at $10 \mathrm{ng} / \mathrm{mL}$ each for 2 hours before cell lysis. The mRNA expression levels were normalized to GAPDH and the values were presented in $2^{-\Delta \Delta \mathrm{CT}}$ as the mean $\pm \mathrm{SD}, n=3,{ }^{*} P<0.05$. Statistics was performed as described in Materials and Methods. (d) A representative western blot analysis of ICAM-1 expression with (+) and without (-) ESE-1-RNAi, compared to that of the C4HSU vector control group on day 4 and day 5 after transduction. Since ICAM-1 levels were very low, subgroup of cells were stimulated with TNF- $\alpha$ and IL-1 $\beta$ (10 ng/mL each) for 16 hours to visualize ICMA-1 protein expression before cell lysis.

airway epithelium in order to alleviate airway inflammatory diseases.

In this study, we show that both ESE-1 and ICAM-1 mRNAs are upregulated in lung epithelial cell lines after treatment with TNF- $\alpha$ and IL-1 $\beta$. However, while both genes can be activated by the NF- $\kappa \mathrm{B}[42]$, we questioned whether there was any regulatory relationship between the two. The regulation of ICAM-1 gene expression is primarily at the transcriptional level by several signaling pathways including protein kinase $\mathrm{C}(\mathrm{PKC})$, mitogen activated protein (MAP) kinase (JNK, ERK, and P38), and NF- $\kappa$ B. The human ICAM-1 gene promoter contains binding sites for many transcription factors, including AP-1, C/EBP, Ets, NF- $\kappa$ B, STAT, and Sp1 [42]. Since ESE-1 is a member of Ets family transcription factors which shares DNA binding sites, we decided to examine whether ESE-1 regulated ICAM-1 expression. To investigate the relationship between these two genes, we used helperdependent adenovirus vector containing shRNA-ESE-1 to knockdown ESE-1. We observed that ICAM-1 expression in A549 cells was indeed decreased after knocking down ESE-1. 


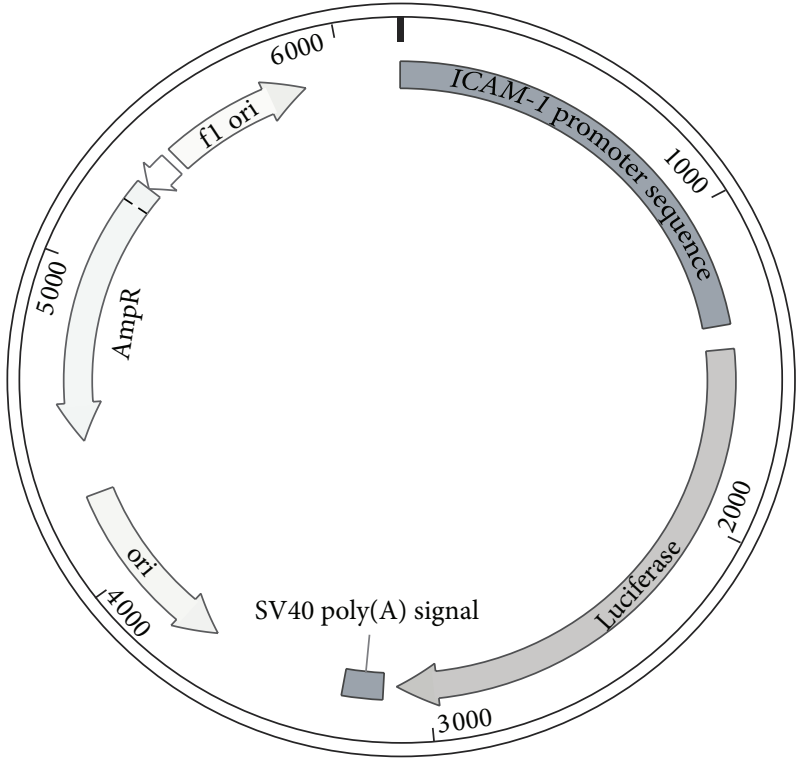

(a)

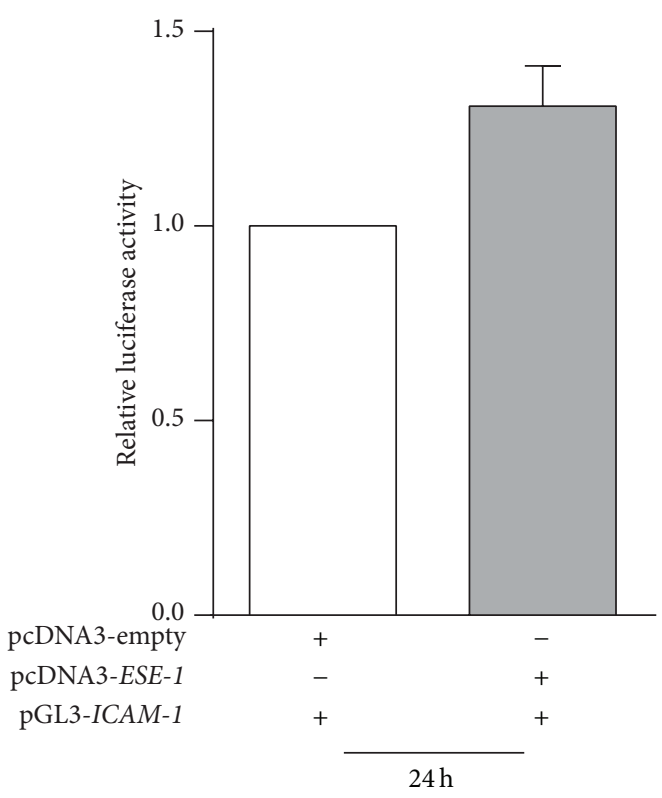

(b)

FIGURE 3: Luciferase assay on BEAS2B cells after cotransfection with pcDNA3-ESE-1 and PGL3-ICAM-1 vector. (a) Schematic diagram of pGL3-ICAM-1 promoter reporter plasmid that contains an ICAM-1 promoter sequence expressing the luciferase gene in the pGL3-Basic vector (Promega). (b) Luciferase activity assay. BEAS-2B cells were cotransfected with pGL3-ICAM-1 luciferase reporter plasmid and pcDNA3-ESE-1 or pcDNA3-empty vector [37]. The luciferase activity was measured $24 \mathrm{~h}$ after transfection.

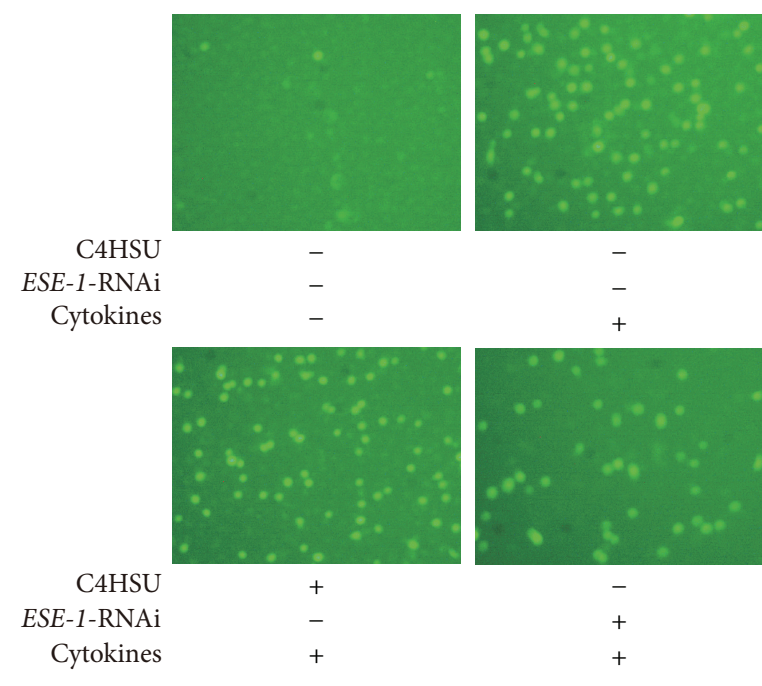

(a)

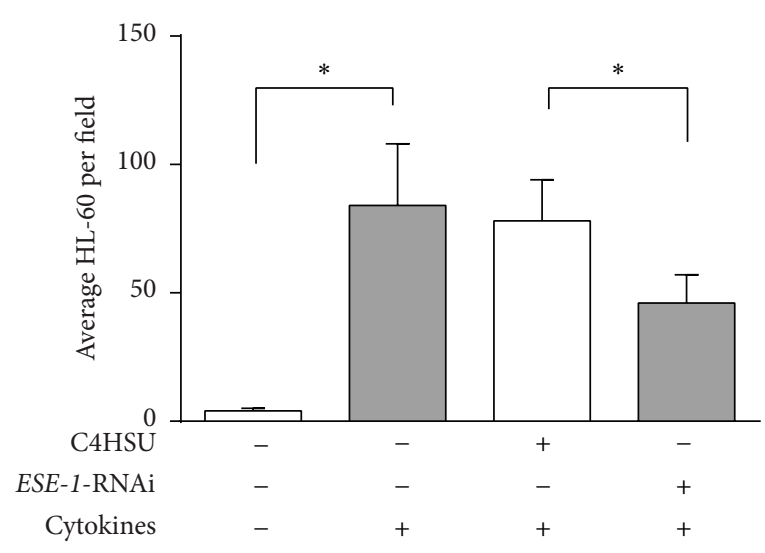

(b)

FIGURE 4: Cell adhesion assay of the effect of ESE-1 knocking down on HL-60 binding to A549 cells. (a) Images showing HL-60 cells attached to A549 cells at 40x magnification. (b) Quantification of cell binding. The average number of HL-60 cells per field was compared among groups with ESE-1 knocking down or C4HSU empty vector transfection or nontransfected groups with or without cytokine stimulation (TNF- $\alpha$ and IL-1 $\beta$ at $10 \mathrm{ng} / \mathrm{mL}$ each). Cell numbers were counted under a microscope and data from six wells were presented as mean \pm SD, ${ }^{*} P<0.05$.

To further investigate on the mechanism of this relationship between ESE-1 and ICAM-1, we used ICAM-1 promoter luciferase reporter assays to determine whether this upregulation was at the transcription level. Upon cotransfecting the luciferase reporter plasmid with an ESE-1 gene expressing plasmid into BEAS-2B cells, we observed an increase in luciferase activity when ESE-1 gene was overexpressed compared to the cells cotransfected with an empty vector plasmid. This result suggests that ESE-1 regulates ICAM-1 expression at the transcriptional level. However, we noted that 
the level of upregulation was not dramatic, but under chronic inflammatory conditions, a small change in ICAM-1 levels may have a significant impact on disease progression.

We also performed a cell adhesion assay to investigate whether knocking down ESE-1 could affect cell adhesion of epithelial cells. We show that, following transduction with ESE-1-RNAi vector, A549 cells exhibited less binding of HL60 cells compared to groups of transduced with C4HSU empty vector or untransduced. This suggests that knocking down ESE-1 in A549 cells causes downregulation of ICAM-1 gene expression which in turn results in a reduced binding interaction between HL-60 and A549 cells.

ICAM-1 is a key molecule that contributes to the control of inflammatory process. Although our finding of ESE-1 regulating ICAM-1 expression in this work is interesting, there are limitations to its clinical applications. First, ESE1 knocking down was carried out only in A549 cells, not in primary CF lung epithelial cells. Second, similar experiments have not been performed in any CF animal model. Future studies on this regulation in other model systems will be important for the development of effective anti-inflammatory strategies suitable for therapeutic intervention of inflammatory conditions such as CF. Since ESE-1 is an transcription factor, it could be a potential drug target for screening small molecules that inhibit its expression.

\section{Conflict of Interests}

The authors declare that there is no conflict of interests regarding the publication of this paper.

\section{Acknowledgments}

The authors would like to thank Rongqi Duan for viral vector production and Anan Wang for technical assistance in cell adhesion assays and transduction experiments. The work was partially supported by funds from the Canadian Institutes of Health Research to Jim Hu. Zhiqi Yu is a scholarship recipient of the Guangzhou Elite Program (GEP). Chan Mi Lee is a recipient of the Sick Kids Restracomp Studentship.

\section{References}

[1] J. M. Pilewski and R. A. Frizzell, "Role of CFTR in airway disease," Physiological Reviews, vol. 79, no. 1, pp. S215-S255, 1999.

[2] J. R. W. Govan and V. Deretic, "Microbial pathogenesis in cystic fibrosis: mucoid Pseudomonas aeruginosa and Burkholderia cepacia," Microbiological Reviews, vol. 60, no. 3, pp. 539-574, 1996.

[3] T. Z. Khan, J. S. Wagener, T. Bost, J. Martinez, F. J. Accurso, and D. W. H. Riches, "Early pulmonary inflammation in infants with cystic fibrosis," The American Journal of Respiratory and Critical Care Medicine, vol. 151, no. 4, pp. 1075-1082, 1995.

[4] T. L. Bonfield, J. R. Panuska, M. W. Konstan et al., "Inflammatory cytokines in cystic fibrosis lungs," American Journal of Respiratory and Critical Care Medicine, vol. 152, no. 6, pp. 21112118, 1995.
[5] J. Li, X. D. Johnson, S. Iazvovskaia, A. Tan, A. Lin, and M. B. Hershenson, "Signaling intermediates required for NF-kappaB activation and IL-8 expression in CF bronchial epithelial cells," The American Journal of Physiology - Lung Cellular and Molecular Physiology, vol. 284, no. 2, pp. L307-L315, 2003.

[6] A. Venkatakrishnan, A. A. Stecenko, G. King et al., "Exaggerated activation of nuclear factor $-\kappa \mathrm{B}$ and altered $\mathrm{I} \kappa \mathrm{B}-\beta$ processing in cystic fibrosis bronchial epithelial cells," The American Journal of Respiratory Cell and Molecular Biology, vol. 23, no. 3, pp. 396403, 2000.

[7] R. M. Strieter, "Interleukin-8: a very important chemokine of the human airway epithelium," The American Journal of Physiology-Lung Cellular and Molecular Physiology, vol. 283, no. 4, pp. L688-L689, 2002.

[8] M. S. Diamond, D. E. Staunton, A. R. de Fougerolles et al., "ICAM-1 (CD54): a counter-receptor for Mac-1 (CD11b/CD18)," Journal of Cell Biology, vol. 111, no. 6, pp. 3129-3139, 1990.

[9] M. S. Diamond, D. E. Staunton, S. D. Marlin, and T. A. Springer, "Binding of the integrin Mac-1 (CD11b/CD18) to the third immunoglobulin-like domain of ICAM-1 (CD54) and its regulation by glycosylation," Cell, vol. 65, no. 6, pp. 961-971, 1991.

[10] B. Beck-Schimmer, R. C. Schimmer, and T. Pasch, "The airway compartment: chambers of secrets," News in Physiological Sciences, vol. 19, no. 3, pp. 129-132, 2004.

[11] E. C. Butcher, "Leukocyte-endothelial cell recognition: three (or more) steps to specificity and diversity," Cell, vol. 67, no. 6, pp. 1033-1036, 1991.

[12] J. C. Kidney and D. Proud, "Neutrophil transmigration across human airway epithelial monolayers: mechanisms and dependence on electrical resistance," The American Journal of Respiratory Cell and Molecular Biology, vol. 23, no. 3, pp. 389-395, 2000 .

[13] L. Liu, F. P. J. Mul, T. W. Kuijpers, R. Lutter, D. Roos, and E. F. Knol, "Neutrophil transmigration across monolayers of endothelial cells and airway epithelial cells is regulated by different mechanisms," Annals of the New York Academy of Sciences, vol. 796, pp. 21-29, 1996.

[14] M.-P. Carreno, N. Chomont, M. D. Kazatchkine et al., "Binding of LFA-1 (CD1la) to intercellular adhesion molecule 3 (ICAM-3; CD50) and ICAM-2 (CD102) triggers transmigration of human immunodeficiency virus type 1-infected monocytes through mucosal epithelial cells," Journal of Virology, vol. 76, no. 1, pp. 32-40, 2002.

[15] Y. van Kooyk and T. B. H. Geijtenbeek, "A novel adhesion pathway that regulates dendritic cell trafficking and $\mathrm{T}$ cell interactions," Immunological Reviews, vol. 186, pp. 47-56, 2002.

[16] Y.-J. Chang, M. J. Holtzman, and C.-C. Chen, "Interferon- $\gamma$ induced epithelial ICAM-1 expression and monocyte adhesion. Involvement of protein kinase c-dependent c-Src tyrosine kinase activation pathway," The Journal of Biological Chemistry, vol. 277, no. 9, pp. 7118-7126, 2002.

[17] J. L. Gaglia, E. A. Greenfield, A. Mattoo, A. H. Sharpe, G. J. Freeman, and V. K. Kuchroo, "Intercellular adhesion molecule 1 is critical for activation of CD28-deficient T cells," Journal of Immunology, vol. 165, no. 11, pp. 6091-6098, 2000.

[18] S. K. Shaw, S. Ma, M. B. Kim et al., "Coordinated redistribution of leukocyte LFA-1 and endothelial cell ICAM-1 accompany neutrophil transmigration," The Journal of Experimental Medicine, vol. 200, no. 12, pp. 1571-1580, 2004. 
[19] K. Somersalo, N. Anikeeva, T. N. Sims et al., "Cytotoxic T lymphocytes form an antigen-independent ring junction," Journal of Clinical Investigation, vol. 113, no. 1, pp. 49-57, 2004.

[20] P. W. Thompson, A. M. Randi, and A. J. Ridley, "Intercellular adhesion molecule (ICAM)-1, but not ICAM-2, activates RhoA and stimulates c-fos and rhoA transcription in endothelial cells," Journal of Immunology, vol. 169, no. 2, pp. 1007-1013, 2002.

[21] A. R. Burns, F. Takei, and C. M. Doerschuk, "Quantitation of ICAM-1 expression in mouse lung during pneumonia," Journal of Immunology, vol. 153, no. 7, pp. 3189-3198, 1994.

[22] J. Guzman, T. Izumi, S. Nagai, and U. Costabelt, "ICAM-1 and integrin expression on isolated human alveolar type II pneumocytes," European Respiratory Journal, vol. 7, no. 4, pp. 736-739, 1994.

[23] B. H. Kang, J. D. Crapo, C. D. Wegner, L. G. Letts, and L. Y. Chang, "Intercellular adhesion molecule-1 expression on the alveolar epithelium and its modification by hyperoxia," The American Journal of Respiratory Cell and Molecular Biology, vol. 9, no. 4, pp. 350-355, 1993.

[24] C. Madjdpour, B. Oertli, U. Ziegler, J. M. Bonvini, T. Pasch, and B. Beck-Schimmer, "Lipopolysaccharide induces functional ICAM-1 expression in rat alveolar epithelial cells in vitro," The American Journal of Physiology - Lung Cellular and Molecular Physiology, vol. 278, no. 3, pp. L572-L579, 2000.

[25] B. Beck-Schimmer, C. Madjdjpour, S. Kneller et al., "Role of alveolar epithelial ICAM-1 in lipopolysaccharide-induced lung inflammation," European Respiratory Journal, vol. 19, no. 6, pp. 1142-1150, 2002.

[26] A. G. Frick, T. D. Joseph, L. Pang, A. M. Rabe, J. W. St. Geme III, and D. C. Look, "Haemophilus influenzae stimulates ICAM-1 expression on respiratory epithelial cells," Journal of Immunology, vol. 164, no. 8, pp. 4185-4196, 2000.

[27] C. Hubeau, M. Lorenzato, J. P. Couetil et al., "Quantitative analysis of inflammatory cells infiltrating the cystic fibrosis airway mucosa," Clinical \& Experimental Immunology, vol. 124, no. 1, pp. 69-76, 2001.

[28] O. Tabary, H. Corvol, E. Boncoeur et al., "Adherence of airway neutrophils and inflammatory response are increased in CF airway epithelial cell-neutrophil interactions," The American Journal of Physiology - Lung Cellular and Molecular Physiology, vol. 290, no. 3, pp. L588-L596, 2006.

[29] P. G. M. Bloemen, M. C. van den Tweel, P. A. J. Henricks et al., "Expression and modulation of adhesion molecules on human bronchial epithelial cells," The American Journal of Respiratory Cell and Molecular Biology, vol. 9, no. 6, pp. 586-593, 1993.

[30] T. M. Krunkosky, B. M. Fischer, L. D. Martin, N. Jones, N. J. Akley, and K. B. Adler, "Effects of TNF- $\alpha$ on expression of ICAM-1 in human airway epithelial cells in vitro: signaling pathways controlling surface and gene expression," The American Journal of Respiratory Cell and Molecular Biology, vol. 22, no. 6 , pp. 685-692, 2000.

[31] C. R. Fakler, B. Wu, H. W. McMicken, R. S. Geske, and S. E. Welty, "Molecular mechanisms of lipopolysaccharide induced ICAM-1 expression in A549 cells," Inflammation Research, vol. 49, no. 2, pp. 63-72, 2000.

[32] T. Oikawa and T. Yamada, "Molecular biology of the Ets family of transcription factors," Gene, vol. 303, no. 1-2, pp. 11-34, 2003.

[33] E. Lelièvre, F. Lionneton, F. Soncin, and B. Vandenbunder, "The Ets family contains transcriptional activators and repressors involved in angiogenesis," International Journal of Biochemistry and Cell Biology, vol. 33, no. 4, pp. 391-407, 2001.
[34] A. Seth and D. K. Watson, "ETS transcription factors and their emerging roles in human cancer," European Journal of Cancer, vol. 41, no. 16, pp. 2462-2478, 2005.

[35] P. Oettgen, R. M. Alani, M. A. Barcinski et al., "Isolation and characterization of a novel epithelium-specific transcription factor, ESE-1, a member of the ets family," Molecular and Cellular Biology, vol. 17, no. 8, pp. 4419-4433, 1997.

[36] J. Wu, R. Duan, H. Cao et al., "Regulation of epitheliumspecific Ets-like factors ESE-1 and ESE-3 in airway epithelial cells: Potential roles in airway inflammation," Cell Research, vol. 18, no. 6, pp. 649-663, 2008.

[37] C. M. Newnham, T. Hall-Pogar, S. Liang et al., "Alternative polyadenylation of MeCP2: influence of cis-acting elements and trans-acting factors," RNA Biology, vol. 7, no. 3, 2010.

[38] K. J. Livak and T. D. Schmittgen, "Analysis of relative gene expression data using real-time quantitative PCR and the $2^{-\triangle \Delta C_{T}}$ method," Methods, vol. 25, no. 4, pp. 402-408, 2001.

[39] H. Takizawa, "Airway epithelial cells as regulators of airway inflammation (Review)," International Journal of Molecular Medicine, vol. 1, no. 2, pp. 367-378, 1998.

[40] N. Aldallal, E. E. McNaughton, L. J. Manzel et al., "Inflammatory response in airway epithelial cells isolated from patients with cystic fibrosis," The American Journal of Respiratory and Critical Care Medicine, vol. 166, no. 9, pp. 1248-1256, 2002.

[41] J. Beale, A. Jayaraman, D. J. Jackson et al., "Rhinovirusinduced IL-25 in asthma exacerbation drives type 2 immunity and allergic pulmonary inflammation," Science Translational Medicine, vol. 6, Article ID 256ra134, 2014.

[42] A. van de Stolpe and P. T. van der Saag, "Intercellular adhesion molecule-1," Journal of Molecular Medicine, vol. 74, no. 1, pp.1333, 1996. 


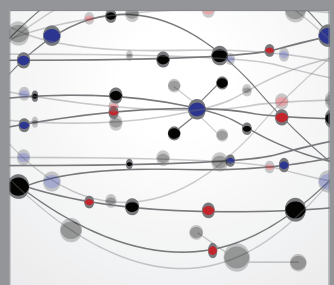

The Scientific World Journal
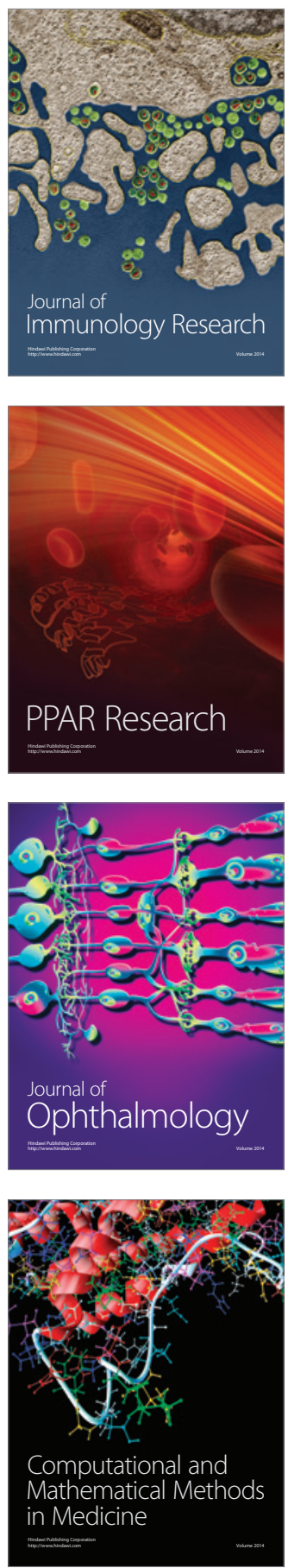

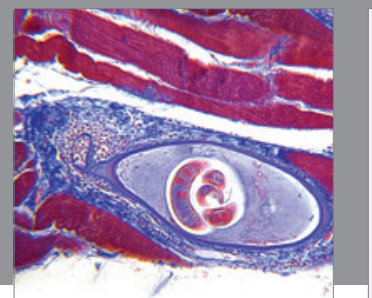

Gastroenterology

Research and Practice
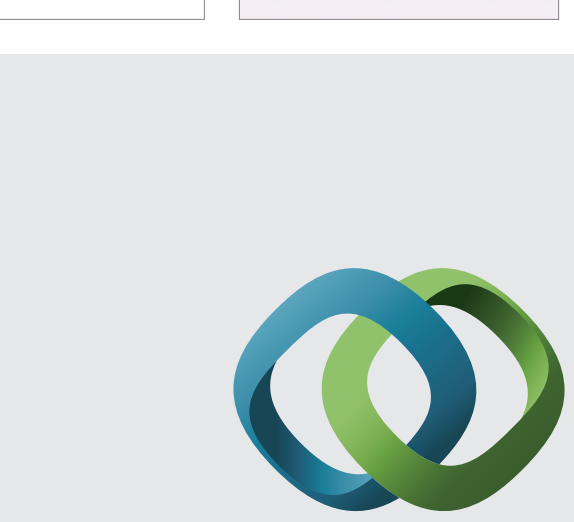

\section{Hindawi}

Submit your manuscripts at

http://www.hindawi.com
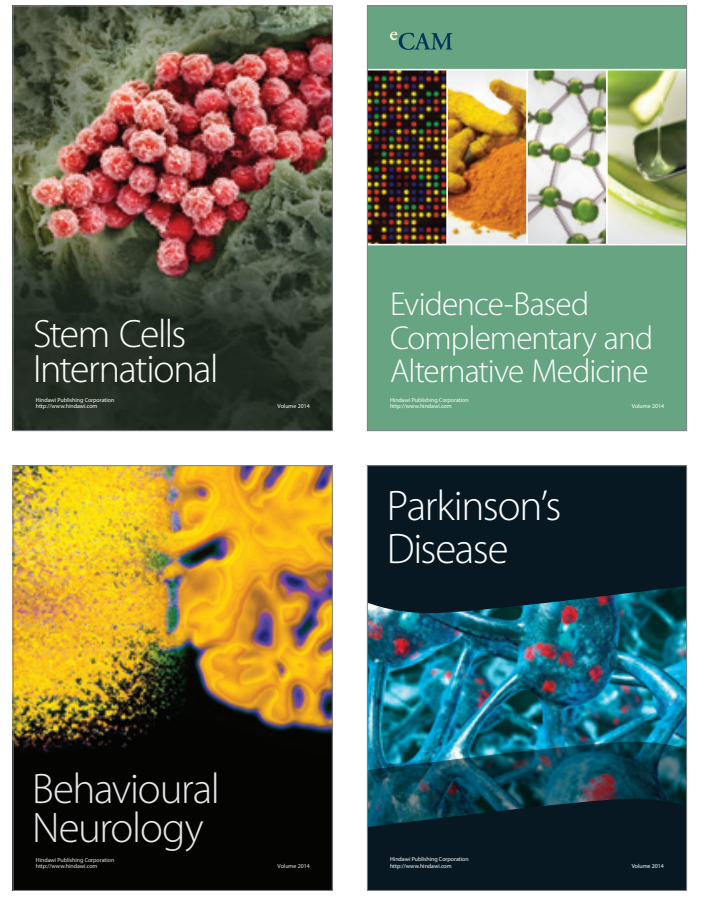
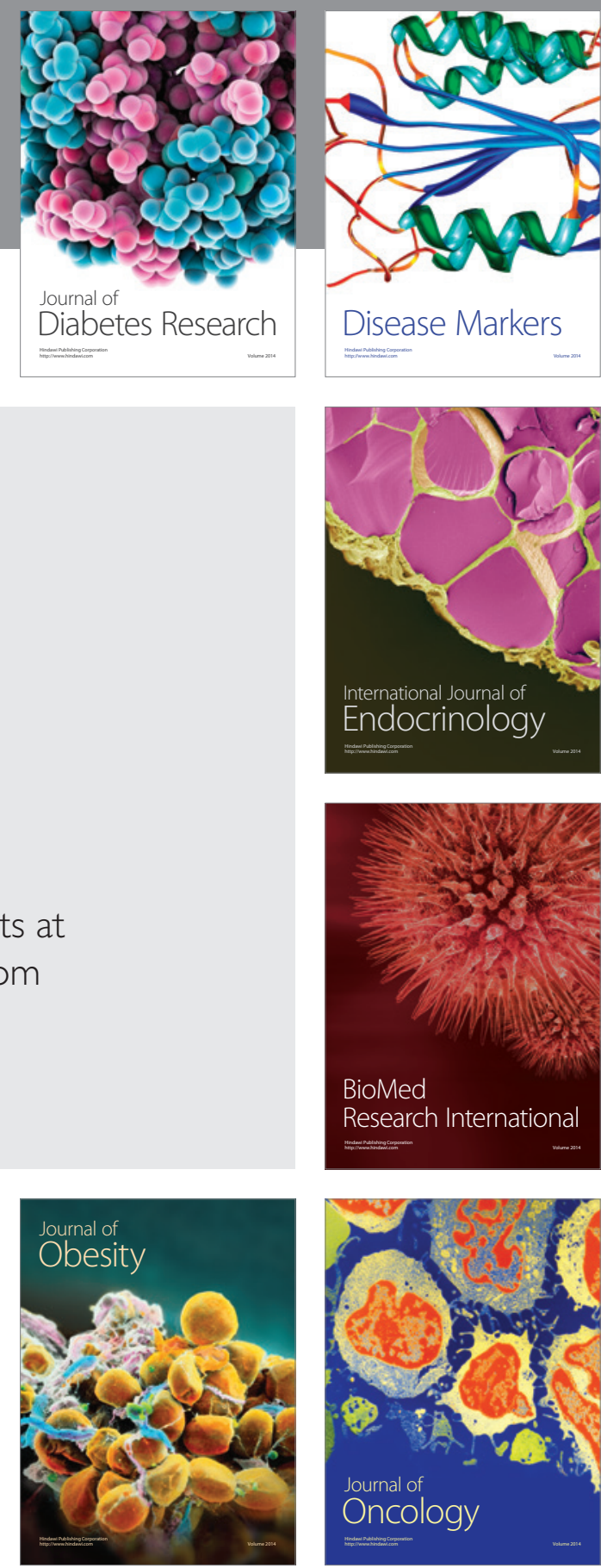

Disease Markers
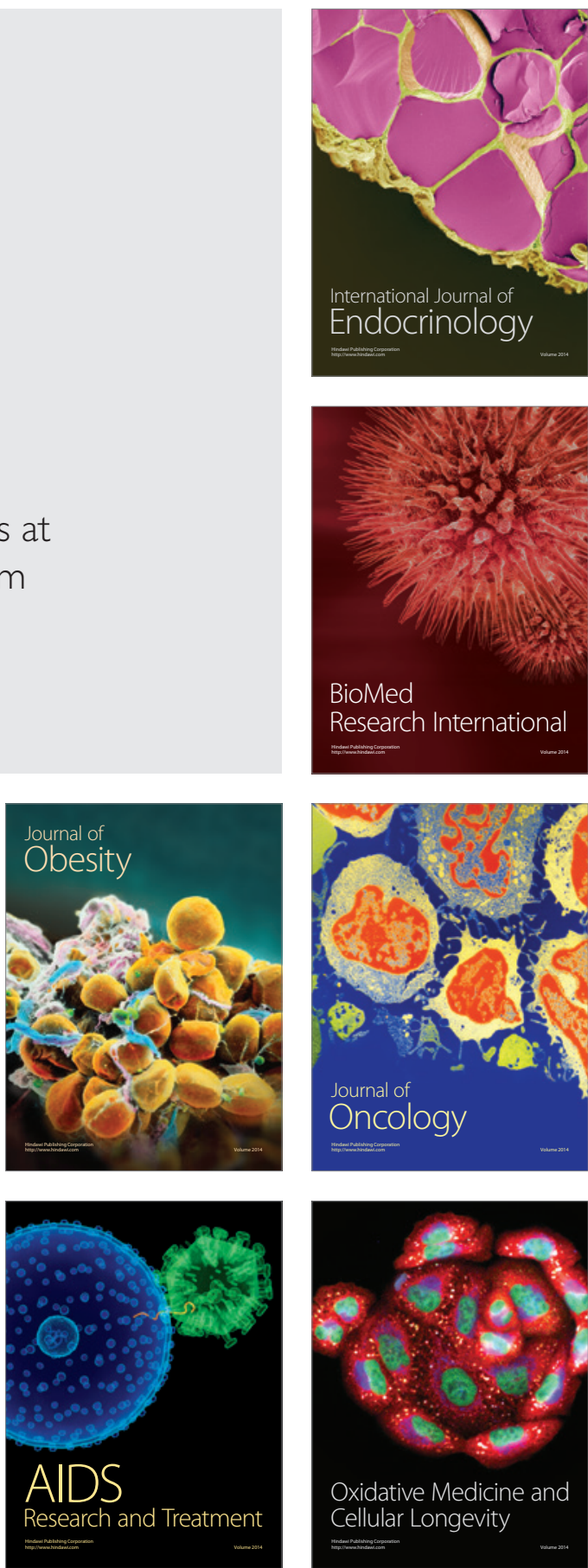